\title{
Understanding Spatial Related Network Challenges from Physical and Network Layers
}

\author{
Cheng Leong Lim ${ }^{12}$, Cindy $\mathrm{Goh}^{2}$, Asiya $\mathrm{Khan}^{3}$, Yun $\mathrm{Li}^{2}$ \\ ${ }^{1}$ NXP Semiconductors, Singapore 138628 \\ ${ }^{2}$ School of Engineering, University of Glasgow, Glasgow G12 8LT, U.K \\ ${ }^{3}$ School of Marine Science \& Engineering, University of Plymouth, Plymouth, Devon, PL4 8AA, UK \\ chengleong.lim@nxp.com, cindy.goh@glasgow.ac.uk, asiya.khan@plymouth.ac.uk, \\ yun.li@glasgow.ac.uk
}

\begin{abstract}
Wireless Sensor Network's communication reliability is greatly influenced by the spatial related network challenges found in the physical space between communicating nodes. In this paper, ZigBee based sensor nodes are experimented under the influence of two distinct spatial related network challenges (i) poor deployed environment and (ii) human movements. WSN parameters obtained are used to develop an ANFIS based model designed to predict these spatial related network challenges. Using ANFIS model prediction accuracies as performance indices, WSN parameters are analysed from the physical and network layers perspective. Physical layer's link properties, reception strength and reception variability, are shown to be key indicators to spatial related network challenges. The parameters observed are Mean RSSI, Average Coefficient of Variation RSSI, Neighbour Table Connectivity and Bidirectional Neighbour Table Connectivity.
\end{abstract}

\section{BACKGROUND}

Wireless Sensor Network's (WSN) communication reliability and a node's operating lifetime are greatly influenced by the network challenges found in the deployed environment [1, 2]. Particularly for indoor WSN applications, spatial related network challenges are known to distort and attenuate transmitted signals [3]. Here, spatial related network challenges are referred as the occurrences in the physical space between communicating nodes that can cause a link to fail. These challenges are, but not limited to, poor deployed environment and human movements.

The propagation of transmitted signals is uniquely determined by the physical medium between nodes; depending on how the nodes are deployed and the dynamics of the environment, it has direct influence on the reliability of wireless communication. Objects in physical medium such as walls and cabinets act as reflectors creating different propagation paths for a transmitted signal [20]. As a result, the receiving node receives superposition of multiple copies of the transmitted signal; each arriving at different times and of different qualities. In addition, the quality of transmitted signals varies with time $[12,13,14]$ - a change in environment such as opening of a door or physical human movement can introduce a temporal alteration in propagation path of a transmitted signal.

WSN optimisation protocols designed to mitigate network challenges are often necessary to reduce the energy footprint and optimise the WSN operating lifetime [7]. Link quality estimation is the fundamental of optimisation protocols [8]. For instance, a typical Transmission Power Control protocol reduces a node's energy consumption by adapting the transmit power according to its link budget and connectivity between communicating nodes [7]. Nonetheless, failure to identify the cause of link failures may have an adverse effect $[1,9]$. For example, increasing transmit power may not be effective for nodes suffering from channel access failure under persistent Wi-Fi interference [10] and may in turn result in an increase in energy consumption. Hence, whilst optimisation protocols can mitigate some network challenges, their performance still boil down to how accurately link quality and network failures can be estimated.

In reality, network challenges such as fading and multipath effects [20] are observed to be random unless the positions of all nodes and the dynamics of the environment are known and uninterrupted at all times. Such information is not practically obtainable in a real world deployment. In addition, network challenges generally influence individual WSN communication layers differently [7, 11, 21]. A topology failure could be potentially attributed to either the poor time synchronisation characteristic of MAC layer or poor end-to-end connectivity characteristic of routing layer. A failure detection mechanism belonging to a specific layer should not be representative without knowing the response from other layers [7]. For these reasons, it is essential to investigate the behavior of parameters from different WSN communication layers under different conditions in order to improve link failure characterisation.

Therefore the intention of this work is to experiment WSN parameters under the influence of spatial related network challenges. Specifically, this paper focuses on parameters from the physical layer and network layer. These parameters are mean RSSI and Average Coefficient of Variation RSSI (ACV RSSI) 
from the physical layer, and Neighbour Table Connectivity (NTC) and Bi-directional Neighbour Table Connectivity (BNTC) from the network layer.

The objectives of this paper are twofold. Firstly, this paper aims to experiment WSN parameters under the influence of two distinct spatial related network challenges: (i) poor deployed environment and (ii) human movements. The obtained WSN parameters are then used as training inputs to an Adaptive Neuro Fuzzy Inference system (ANFIS) [20] model. Secondly, the trained ANFIS model's prediction accuracies are used as performance indicators, where the importance of parameters under study are compared and ranked.

The remainder of this paper is organised as follows: Section II reviews the related research work pertaining to spatial related network challenges. Section III explains the WSN parameters to be studied, while section IV describes the experimental setups. Section V explains the basis of ANFIS and also proposes three network conditions to be modeled. The results of the ANFIS model are discussed and summarized in Section VI and Section VII respectively. Lastly, Section VIII concludes the paper with key learning made.

\section{RELATED WORKS}

In [16], the impact of human's physical obstructions on $2.4 \mathrm{GHz}$ wireless signals are studied and showed that the number of people around a node and their movement speed directly correlate to the variation of RSSI measurements. The impact of antenna orientation, elevation, and ground effect on wireless communication are studied in [17]. It is also observed that even without human movements, RSSI measurements are inconsistent at different nodes position. In [18], the influence of building layout on communicating nodes was measured with signal attenuation and communication success rate. It has shown that a node found suffering from poor deployment can be predicted from measures of quality of services and overall energy consumption. In [19], signal deviation and path loss are found to vary with locations, i.e. building structures such as corridors, walls, and adjacent floors have inconsistent impact on signal propagation, resulting in different measurements obtained at similar locations.

In [4], reception rate and link asymmetry are found to vary in different environments. Empirical studies [1, 24] on wireless sensor networks have shown that radio quality and communication range vary with directions resulting in an irregular pattern of coverage and communication. Another aspect of link quality is its temporal characteristics, where link quality varies with the time. In [12] the change of signal to noise ratio with time is studied, and $\beta$-factor, a metric to measure link "burstiness", is introduced. $\beta$-factor, the probability of a link switches between good and bad delivery, is used to calculate retransmission interval after encountering a packet failure.

A study in [14] confirms the presence of a "grey region" in indoor office, outdoor habitat, and an open parking lot where similar RSSI samples may represent a wide range of PRR values. This phenomenon is explained with nodes with shorter communication range may reduce the chances of multipath and improve packet delivery. An RSSI-distance estimation technique is developed in [22]. To improve the overall distance estimation accuracy, multi path, shadowing effect, and interference from other RF sources, are accounted for as an environmental error. A metric consisting of LQI, RSSI and PRR is developed in [23] to access link quality in a both static and mobile network. It is observed that the shorter a link quality observation window is, the noisier these hardware indicators are. A thorough study of all available parameters is recommended before utilising them.

\section{PARAMETERS}

In this work, the parameters to study are extracted from the ZigBee PRO nodes' Neighbour Table (NT) [5]. A node's NT contains connectivity information about its neighbors within receptive range, including relative RSSI. If node A is not present in node B's NT, node A's incoming signal is simply not received by node $\mathrm{B}$ at the moment of reported NT. In every experiment conducted (refer to Section IV), experimental ZigBee PRO nodes regularly report information of their NT to a sink. Depending on the network size, the reporting intervals vary between 4-6 seconds. By observing multiple NTs over a period of time, the following parameters are processed.

\section{A. Network layer parameter}

\section{1) Neighbour Table Connectivity}

Table I illustrates how Neighbour Table Connectivity (NTC) is calculated between two nodes. $\mathrm{NTC}_{\mathrm{AB}}$, similar to Packet Reception Rate, is the probability of node $\mathrm{B}$ being captured in node A's NT observed over a span of 60 seconds. $N T C_{A B}$, indicates how consistent node $\mathrm{B}$ is connected to node $\mathrm{A}$ in percentage.

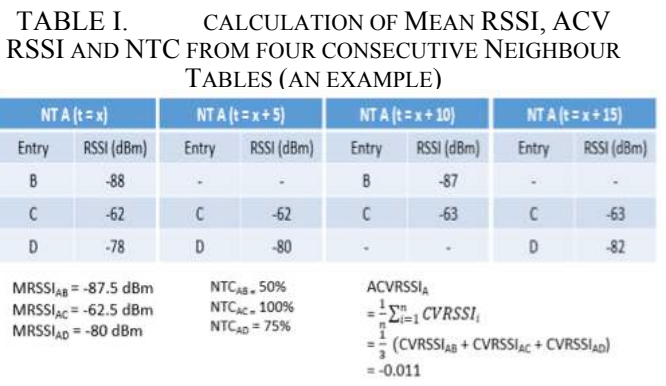




\section{2) Bi-direction Neighbour Table} Connectivity (BNTC)

Similar to NTC, Bi-NTC can be explained as the probability of connectivity between two nodes. Both $\mathrm{BNTC}_{\mathrm{AB}}$ and $\mathrm{NTC}_{\mathrm{AB}}$ represent the out-going and incoming communication success rate between nodes $\mathrm{A}$ and $\mathrm{B}$ respectively, and are both calculated in the same manner. In other words, $\mathrm{BNTC}_{\mathrm{AB}}$ is essentially $\mathrm{NTC}_{\mathrm{BA}}$. Combining both NTC and BNTC indicate the possibility of link asymmetry between two nodes.

\section{B. Physical layer:}

\section{1) Mean RSSI}

$M_{R S S I} I_{A B}$ as illustrated in Table I is the averaged RSSI in $\mathrm{dBm}$ measured at node $\mathrm{A}$ from node $\mathrm{B}$ over the period of $60 \mathrm{~s}$. MRSSI indicates how well node $\mathrm{B}$ is received by node A in terms of signal strength over an observed window.

2) Average Coefficient of Variation RSSI (ACV RSSI)

$A C V R S S I_{A}$ as calculated in Table $\mathrm{I}$, is the average coefficient of variation of RSSI in $\mathrm{dBm}$ of all links found in node A's NT. ACVRSSI can be explained as a measure of reception signal dispersion between all neighbouring nodes around node $\mathrm{A}$ over an observed window. The calculation of $\mathrm{ACVRSSI}_{\mathrm{A}}$ is expressed as:

$$
\operatorname{ACVRSSI}_{A}=\frac{1}{n} \sum_{i=1}^{n} \frac{\operatorname{SDRSSI}_{A i}}{\operatorname{MRSSI}_{A i}}
$$

where $n$ is the number of neighbouring nodes around node A, while SDRSSI is the standard deviation of RSSI over the same observed window.

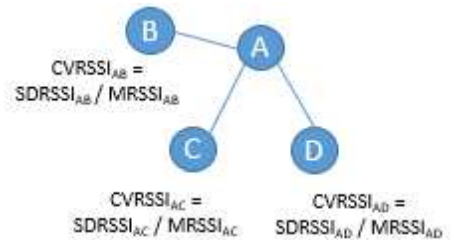

\section{EXPERIMENTAL SETUPS}

Two separate experiments as described in the following sections are prescriptively controlled and conducted during non-working hours in a static office environment to simulate link failures influenced by (i) poor deployed environment or (ii) human movement.

\section{A. Experiment 1: Poor Deployed Environment}

Experiment 1, as illustrated in Figure 1 consists of four ZigBee PRO [6] nodes uniformly deployed in an open office environment $5 \mathrm{~m}$ apart from one another. The intention of experimental layout is to simulate nodes communicating under the influence of reception signal decay and increasing signal attenuation, which mimics long distance communication and dense environment respectively. All nodes were mounted in the same orientation and at the height of $1.3 \mathrm{~m}$ on desk partitions. It is important to note that due to the difference in physical environment (i.e. desk), a uniform RSSI decay between nodes should not be expected. These differences shall provide a more realistic set of measurements as compared to data obtained from computer simulations.

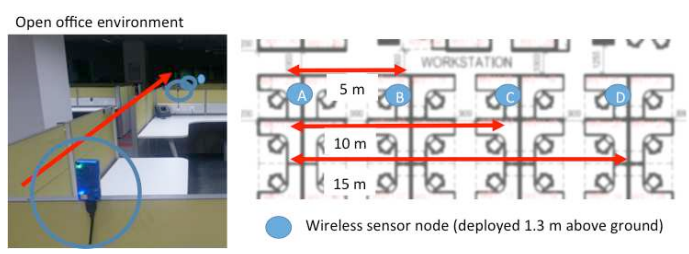

Figure 1. Experiment layout - Poor deployed environment in an open office

\section{B. Experiment 2: Human Movement}

Experiment 2 as shown in Figure 2 consists of two ZigBee PRO nodes [6] deployed $4.5 \mathrm{~m}$ apart with line of sight (LOS) communication. The nodes were mounted on tripods where distance from ground was elevated to $1.3 \mathrm{~m}$. The design of experiment is to simulate nodes communicating under the influence of single human movement profile. Prescribed human walking sequences with LOS obstruction were introduced during parts of the experiments. In addition, experiment 2 was repeated in an empty laboratory and open office where different multipath and fading effects were accounted for, providing a more realistic and representative measurements for ANFIS model training.

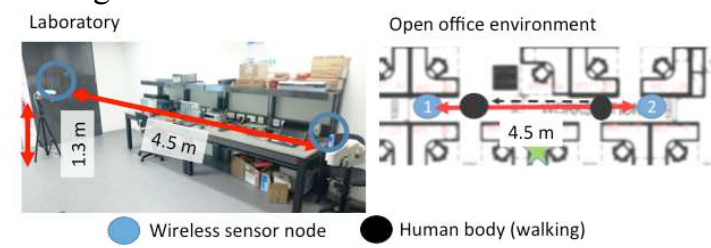

Figure 2. Experiment layout - Human movement in a laboratory and open office

\section{ADAPTIVE NEURO-FUZZY INFERENCE SYSTEM}

ANFIS [15] combines the approximate reasoning of fuzzy set theory with the learning features of Artificial Neural Networks (ANN) into a single data learning technique. Functionally, ANFIS constructs an input-output mapping in the form of IF-THEN rules, which are interconnected with Neural Network nodes and connections. Due to its ability to handle non-linear systems, ANFIS is used in this work with the intent to break down the conflicting uncertainties found in WSN into comprehensible knowledge.

Figure 3 illustrates a functional block diagram of the ANFIS based model where 
physical and network layer parameters are obtained from experimented nodes, and are used as training inputs for the prediction model. The following three conditions are considered:

- Link Failure Due to Poor Deployed Environmnet: Link failures that are influenced by either dense physical obstructions between nodes or nodes located too far apart.

- Link Failure Due to Human Movements: Link failures that are influenced by physical presence of a human in close proximity.

- No Failure: Best case scenario where a node is not subjected to any interference and any failures are considered negligible.

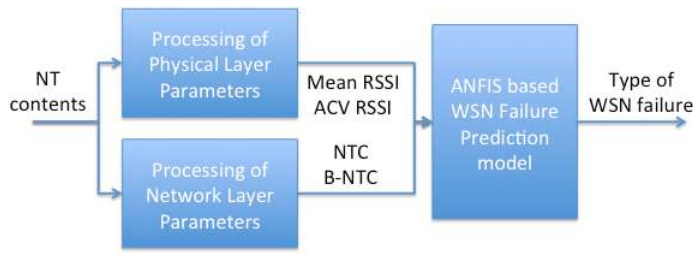

Figure 3. Block diagram of ANFIS-based prediction model

\section{RESULTS AND DISCUSSION}

In this section, the findings from the ANFIS-based model described in Section V are presented. ANFIS model prediction accuracies are used as a performance index to investigate the individual and combined effects of WSN physical and network layer parameters. Model is analysed and explained in-depth with the help of box plots diagram.

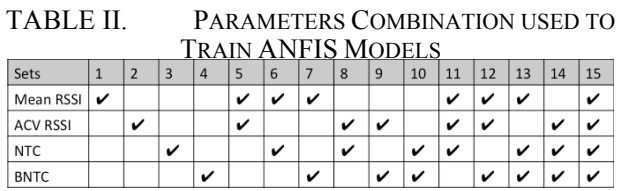

The ANFIS model is trained repeatedly using 15 different sets of input parameters as presented in Table II. The purpose is to better understand the behaviour of different inputs or their combinations thereof under different link failures, so to ascertain which layer has a greater impact on the respective conditions.

\section{A. Comparison between combination sets with 1 parameter}

This section presents the comparison and analysis among single parameter combination sets (set $1-4$ ). Referring to Figure 4, it is clear that mean RSSI (set 1) as single parameter performed significantly better than ACV RSSI, NTC, and BNTC (Sets $2-4$ ) with a prediction accuracy of $81.8 \%$. This suggests that mean RSSI represents the reception strength is suitable for link failure detection caused by spatial related network challenges. A probable explanation for this is that obstructions in the physical medium such as walking human, walls, and desk partitions can influence the transmitted signals via attenuation or distortion, which ultimately degrades RSSI measurements leading to a higher chance of link failure [16, $20]$.

ACV RSSI (Set 2) has the worst performance with an accuracy of only $6.5 \%$. It is observed that under the conditions of "no failure" and "failure due to poor deployed environment (experiment 1) only", multipath and fading effects are subjected to minimal variations due to the static experimental environment. From the experimental results involving static environments, mean RSSI between nodes are found to be relatively constant throughout the experiments with no more than $1.1 \mathrm{dBm}$ variation. This explanation mirrors the poor performance of ACV RSSI that represents reception fluctuation.

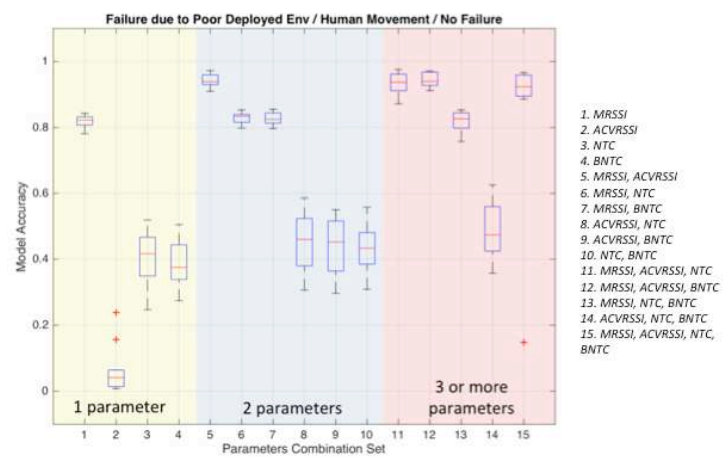

Figure 4. ANFIS prediction results - Link failure due to Poor Deployed Environment vs. Human Movement vs. No Failure.

On the other hand, network layer parameters, NTC and BNTC (Sets 3 and 4) as single parameter produced a relatively poor prediction accuracy of only $40.4 \%$ and $39.2 \%$ respectively. NTC and BNTC representing the connectivity between communicating nodes, are capable of differentiating a link with and without failure, however are insufficient to distinguish between human movements and poor deployed environment (further explained in B).

B. Comparison between combination sets with 2 parameters

In this section, the mean prediction accuracies of combination sets with two parameters (Sets $5-10$ ) in Figure 4 are compared. It is trivial that any parameter combination sets containing mean RSSI (Sets 5 - 7) performed well, producing average accuracies greater than $80 \%$. This is also highlighted in previous section where mean RSSI as a single parameter performed well. It is observed that the prediction accuracy of mean RSSI improved with ACV RSSI (Set 5) compared to with NTC or BNTC (Sets 6 or 7). 
This phenomenon can be explained that obstructions in the physical medium degrades reception strength measurements, while the introduction of human movements inflict temporal changes to multipath and fading effects. Mean RSSI and ACV RSSI represent reception strength and reception fluctuation respectively. The combination of both physical layer parameters not only improved the detection of link failures, but is also capable of distinguishing the cause of failure due to human movements or poor deployed environment with an accuracy of $94.2 \%$.

Looking in-depth into the ANFIS model training samples with mean RSSI poorer than $86 \mathrm{dBm}$ (300 samples), links with NTC and BNTC poorer than $80 \%$ take up only $59 \%$ and $51 \%$ of the entire population respectively. This observation suggests trend towards false positives where NTC and BNTC may wrongly indicate the presence of a particular condition. The inconsistency prediction can be explained by considering links operating at reception sensitivity edge, which may not always lead to link failure. As a result, NTC and BNTC representing connection success rate, and link asymmetry performed poorly when it comes to detecting spatial related network challenges.

C. Comparison between combination sets with 3 or 4 parameters

In this section, the mean prediction accuracies of combination sets with three or more parameters (sets $11-15$ ) in Figure 4 are compared. Once again, it is observed from sets $11-13$ and 15 that combination sets that contain mean RSSI produced good prediction accuracies greater than $80 \%$. Amongst them, sets that contain ACV RSSI (sets 11, 12 and 15) performed even better producing prediction accuracies of more $90 \%$. Clearly, this suggests that poor reception strength and high reception fluctuation (both captured on physical layers) are key contributions to a link failure caused by spatial related network challenges.

Comparison of set 15 (highest number of parameters of 4) with combination sets 5, 11 and 12 (less parameters yet similar prediction accuracies), having more parameters does not necessarily translate to a better prediction accuracy. In other words, adding more training inputs may not necessary produce a more accurate prediction model.

\section{COMPARISON OF THE PARAMETER COMBINATION SETS}

Table III summarises the results for all 15 sets of parameter combinations and are ranked according to the mean prediction accuracies from most to least accurate.
TABLE III. SET OF PARAMETER COMBINATIONS RANKED ACCORDING TO MEAN PREDICTION ACCURACIES

\begin{tabular}{|c|c|c|c|}
\hline$S / N$ & Combi Set & Parameters Used & Mean Accuracy (\%) \\
\hline 1 & 12 & Mean RSSI, ACV RSSI, BNTC & 94.5 \\
\hline 2 & 5 & Mean RSSI, ACV RSSI & 94.3 \\
\hline 3 & 11 & Mean RSSI, ACV RSSI, NTC & 93.5 \\
\hline 4 & 15 & Mean RSSI, ACV RSSI, NTC, BNTC & 85.3 \\
\hline 5 & 6 & Mean RSSI, NTC & 82.9 \\
\hline 6 & 7 & Mean RSSI, BNTC & 82.7 \\
\hline 7 & 1 & Mean RSSI & 81.9 \\
\hline 8 & 13 & Mean RSSI, NTC, BNTC & 81.8 \\
\hline 9 & 14 & ACV RSSI, NTC, BNTC & 48.6 \\
\hline 10 & 8 & ACV RSSI, NTC & 45.4 \\
\hline 11 & 10 & NTC, BNTC & 43.6 \\
\hline 12 & 9 & ACV RSSI, BNTC & 43.5 \\
\hline 13 & 3 & NTC & 40.4 \\
\hline 14 & 4 & BNTC & 39.2 \\
\hline 15 & 2 & ACV RSSI & 6.6 \\
\hline
\end{tabular}

\section{A. Physical layer}

From Table III, it is clear that the mean RSSI stands out among all parameters where the top 8 models all contain mean RSSI as one of their training inputs. Mean RSSI, used on its own or as a joint parameter, is a strong influencer when it comes to detecting link failures due to poor deployed environment ACV RSSI on the other hand appears in the top 4 best predictors. ACV RSSI as a single parameter performed poorly. This could be attributed to minimal variations of multipath and fading effects in a static environment, leading to poor detectability to link failure. However, ACV RSSI when combined with other parameters showed improved overall prediction accuracy. For example, prediction accuracy of "mean RSSI \& BNTC" improved from $82.7 \%$ to $94.5 \%$ with the addition of ACV RSSI). In this work, ACV RSSI represents reception fluctuation, is poor at detecting link failures, but it is still useful where it comes to differentiating link failures between poor deployed environment and human movement.

\section{B. Network layer}

NTC and BNTC as single parameters, and as a joint parameter represent the link connectivity and link asymmetry. All of which are found to be insufficient to predict link failures caused by spatial related network challenges. Here, the poor performances of application layer parameters (NTC and BNTC) are explained to produce false positive prediction and have wrongly indicated the presence of a condition. It is also observed that only under the conditions where mean RSSI is not present, network layer parameters are found to be useful (i.e. prediction accuracy of set 2 
(ACV RSSI) improve from $6.6 \%$ to $48.6 \%$ with addition of NTC and BNTC). Again, this phenomenon suggests more evidence that network parameters are useful at detecting a link failure but not distinguish between failures caused by human movements and poor deployed environment.

\section{CONCLUSION}

In this work, an ANFIS model is developed to predict link failures due to (i) poor deployed environment and (ii) human movements. Findings are then used to inform the impact of WSN parameters from the physical (mean RSSI and ACV RSSI) and network (NTC and BNTC) layers perspectives. Physical layer parameters showed promising results at predicting link failures caused by spatial related network challenges compared to application layer parameters. It is found that Mean RSSI and ACV RSSI are able to predict link failures with better accuracies as compared to NTC and BNTC. Furthermore NTC and BNTC produced false positive predictions trends mirrors the "grey region" observed in [14].

It can be inferred that reception strength and reception variability are critical link properties to distinguish link failures between poor deployed environment and human movement. Individual parameters have shown to behave differently under different conditions. Parameters that performed poorly in this work may be still applicable in different complex environment (i.e. RF interference [10]). Hence, in order to produce an accurate prediction model, it is of great importance to first understand the operating behaviour of parameters under different network challenges, followed by a careful selection of parameters.

\section{REFERENCES}

1] D.Ganesan, D.Estrin, A.Woo, and D.Culler, "Complex Behaviour at Scale: An Experimental Study of Low-Power Wireless Sensor Networks", Vol. 13. Technical Report UCLA/CSD-TR 02, 2002.

[2] N.Baccour, A.Koubaa, M.B.Jamma, D.Rasario, H.Youssef, M.Alves, and L.B.Becker, "RadiaLE: A Framework for Designing and Assessing Link Quality Estimators in Wireless Sensor Networks", Ad Hoc Networks 9, pp. 1165-1185, 2011.

[3] J.Nazabal, P.Iturri, L.Azpilicueta, F.Falcone, and C.Valdivielso, "Performance Analysis of IEEE 802.15.4 Compliant Wireless Devices for Heterogeneous Indoor Home Automation Environment", Journal of Antennas and Propagation, Vol 2012, 2012

[4] D. E. Alberto Cerpa, and Naim Busek, "Scale: A Tool for Connectivity Assessment in Lossy Environments," Technical Report, 2003.

[5] "ZigBee PRO Stack User Guide", NXP Laboratories UK, 2014.

[6] "JN5168-001-Myy Datasheet", NXP Laboratories UK, 2013.

[7] S.Aslam, F. Farooq, and Shahzad Sarwar, "Power Consumption in Wireless Sensor Networks, General Literature", In 6th FIT, 2009.
[8] A.Woo and D.Culler, "Evaluation of Efficient Link Reliability Estimators for Low-Power Wireless Networks," EECS Department, University of California, Berkeley, Tech. Rep. UCB/CSD-03-1270, 2003.

[9] Wireless Sensor Networks: Signal Processing and Communications Perspectives. John Wiley \& Sons, pp. 69-344, 2007.

[10] C.L.Lim, M.Bolt, A.Syed, P.Ng, C.Goh, and Y.Li, "Dynamic Performance of IEEE 802.15.4 Devices under Persistent WiFi Traffic", RIoT, pp. 1-6, 2015.

[11] Su, Weilian, and Tat L. Lim, "Cross-Layer Design and Optimisation for Wireless Sensor Networks", International Journal of Sensor Networks 6.1, 2009.

[12] K.Srinivasan, M.A.Kazandjieva, S.Agarwal, and P.Levis, "The $\beta$-factor: Measuring Wireless Link Burstiness," in Proceedings of the 6th ACM conference on Embedded network sensor systems, SenSys '08. New York, USA: ACM, 2008.

[13] Bas, C.Umit, and S.C.Ergen. "Spatio-Temporal Characteristics of Link Quality in Wireless Sensor Networks", IEEE Wireless Communications and Networking Conference (WCNC), 2012.

[14] Zhao, and R. Govindan, "Understanding Packet Delivery Performance in Dense Wireless Sensor Networks", In Proceedings of the First International Conference on Embedded Network Sensor Systems, 2003.

[15] Jang, and Jyh-Shing Roger. "ANFIS: Adaptive Network-based Fuzzy Inference System", Systems, Man and Cybernetics, IEEE Transactions on 23.3, pp. 665-685, 1993.

[16] M.F.Ramli, L.M.Kamarudin, A.Zakaria, A.Y.M.Shakaff, D.L.Ndzi, C.M.Nor, N.Hassan, and S.Mamduh, "The Study of Human Movement Effect on Signal Strength for Indoor Wireless Sensor Network Deployment", ICWISE, pp 30-35, 2013.

[17] S.Ahmed, S.Bouk, N.Javaid, and I.Sasase, "Combined Human, Antenna Orientation in Elevation Direction and Ground Effect on RSSI in Wireless Sensor Network", CSNI, 2012.

[18] I.Chatzigiannakis, G.Mylonas, and S.Nikoletseas, "Modelling and Evaluation of the Effect of Obstacles on the Performance of Wireless Sensor Network", ANSS'06, 2006.

[19] B. R. Jadhavar, and T. R. Sontakke, " $2.4 \mathrm{GHz}$ Propagation Prediction Models for Indoor Wireless Communication within Building", IJSCE, Vol 2, 2012.

[20] M.Takai, J. Martin, and R.Bagrodia, "Effects of Wireless Physical Layer Modeling in Mobile Ad Hoc Networks', in MobiHoc '01. ACM, 2001.

[21] B.Pavkovic, M.Batic, N.Tomasevic, "The Importance of Cross-layer Considerations in a Standardized WSN Protocol Stack Aiming for IoT", Magazine Ubiquity, Vol 2015.

[22] Bekcibasi, Ugur, and M.Tenruh. "Increasing RSS Localization Accuracy with Distance Reference Anchor in Wireless Sensor Networks." Acta Polytechnica Hungarica 11.8, 2014.

[23] C.Boano, M.Ziga, T.Voigt, A.Willig, and K.Romer, "The Triangle Metric: Fast Link Quality Estimation for Mobile Wireless Sensor Networks," in ICCCN, 2010 Proceedings of 19th International Conference, 2010.

[24] G.Zhou, T.He, S.Krishnamurthy, and J.A.Stankovic, "Impact of Radio Irregularity on Wireless Sensor Networks," in MobiSys '04, USA, 2004. 